\title{
The situational effects on haptic perception of rod length
}

\author{
TIN-CHEUNG CHAN \\ The Chinese University of Hong Kong, Hong Kong
}

\begin{abstract}
Three experiments were conducted to investigate situational effects (manipulation, range, and prior experience) on the haptic perception of rod length. Each rod was held between its two ends with one hand. In Experiment 1, 32 participants judged length of rods using different manipulations. Perceived lengths were found to be dependent on manner of manipulation and not necessarily equal to actual lengths. Different parameters were detected in different manipulations. In Experiment 2, 8 participants judged rod lengths by wielding rods of two ranges: long and short. Perceived length was found to be affected by the range of rods evaluated successively in a single set. In Experiment 3, 9 participants judged rod lengths after an experience of handling dense or light rods. Perceived length was found to be affected by prior experience. Results are discussed in terms of how rod lengths can be perceived accurately by haptic modality without involving direct perception.
\end{abstract}

Haptic perception of rod length has been shown to be dependent on the detection of other properties of the rod. This observation has been claimed to demonstrate information-perception specification: Perception is specific to information, and information is specific to the environment (Gibson, 1979; Kugler \& Turvey, 1987). In vision, light reflected by the environment, called ambient light, carries with it the spatial and temporal patterning that can specify the layout of the environment. In the case of haptic perception of rod length by wielding, the changing flux of stimulation in the muscles and tendons in wielding the rod provides a patterning that specifies the length of the rod (Gibson, 1966).

Previous results have shown that participants use different properties for length specification, depending on the manner of manipulation. Three parameters are involved in specifying length. When a rod is held at the center of mass and kept stationary, weight is the main predictor (Burton \& Turvey, 1990); when the rod is held stationary at one end, static torque (see Appendix for details) is the main predictor (Carello, Fitzpatrick, Domaniewicz, Chan, \& Turvey, 1992) ${ }^{1}$; when the rod is wielded at one end, the moment of inertia is the main predictor for length (Solomon \& Turvey, 1988).

In fact, each of the above three manipulations determines which parameter varies with the testing conditions. When the rod is held stationary at the center of mass, with static torque and moment of inertia equal to zero, only

Preparation of this article was supported by the Shaw College Student Campus Work Scheme (1994 fall) of The Chinese University of Hong Kong. The author would like to thank M. H. Bond for proofreading the first draft, and M. L. Braunstein, S. J. Lederman, and two anonymous reviewers for constructive criticism. Correspondence should be addressed to T.-C. Chan, Department of Psychology, The Chinese University of Hong Kong, Shatin, New Territories, Hong Kong (e-mail: tcchan@cuhk.hk; fax: 852 6035019). weight varies with the testing conditions. When a rod is held horizontally at one end with a mass attached to the rod at different positions, the moment of inertia is zero and weight is kept constant, and thus only static torque varies with the testing conditions. When a rod is wielded at one end with a mass attached at different positions, weight is kept constant, but static torque and moment of inertia change simultaneously with the testing conditions. In each of the above cases, when a certain parameter varies with the testing conditions, it is a good predictor of perceived length. Because the manipulation varies from situation to situation, it can be called a situational factor.

In accordance with Stevens's power law, the mean estimate of a given attribute $\psi(\lambda)$, such as the perceived length, is a power function of the intensity $x$ of a detected physical variable exhibiting that attribute: $\psi(\lambda)=b x^{p}$ where $b$ and $p$ are two scaling coefficients. The power coefficient $p$ is usually regarded as the parameter that characterizes the perceptual continuum (Wyszecki, 1986). Results of previous research on haptic perception of rod length suggest that both scaling coefficients vary according to situations. This is particularly clear with moment of inertia (an inertial property of a wielded rod; see Appendix) treated as the detected physical variable, and its magnitude corresponds to $x$ in the power law. With the moment of inertia, $I_{\mathrm{xx}}$ about the axis of rotation (through $o$ and parallel to the $x$-axis in Figure 4) as the detected parameter, Solomon and Turvey (1988) showed that when a rod is wielded at one end, $b$ varies from 1.68 to 1.86 and $p$ varies from 0.3 to 0.4 . However, when a rod is held between its ends, $b=1.6$, and $p=0.21$. This variation does not vanish when both $I_{1}$ (the diagonalized $I_{\mathrm{xx}}$ ) and $I_{3}$ (the diagonalized $I_{\mathrm{yy}}$, the moment of inertia about the axis of the rod, the $y$-axis in Figure 4) were used as predictors. In three different experiments, Fitzpatrick, Carello, and Turvey (1994) obtained three different equations mapping $I_{1}$ and $l_{3}$ to perceived length, $L_{\mathrm{p}}: L_{\mathrm{p}}=4.74\left(I_{1}^{0.36} I_{3}^{-0.25}\right)$, 
$L_{\mathrm{p}}=2.15\left(I_{1}^{0.37} I_{3}^{-0.17}\right)$, and $L_{\mathrm{p}}=1.03\left(I_{1}^{0.54} I_{3}^{-0.34}\right)$. Although wielding was used exclusively, the scaling coefficients were affected by the experimental conditions. Although no systematic variations can be discerned, the scaling coefficients are clearly situation dependent.

Conversely, rather than a source of error, the change of the scaling coefficients may provide corrections in the haptic perception of rod length. Previously, it has been shown that, with unfamiliar rods, actual rod length cannot be perceived accurately (Chan, 1995). The variables detected, such as weight, static torque, and moment of inertia, are not necessarily proportional to the actual length of rods. As an example, denser rods have larger moments of inertia than lighter rods for the same actual length. Thus, mapping of detected variable to actual length is possible only if the coefficients in the power law covary with the spurious increase or decrease of the detected variable. It is possible that, through recalibration in learning, dense rods with large moments of inertia can be fitted with small scaling coefficients and light rods can be filled with large ones. ${ }^{2}$ With the change of scaling coefficients, perceived length can be made close to actual length. Yet, such variability of the scaling coefficients causes errors in length judgments with unfamiliar rods. Particularly, I propose that when a rod of a specific length and mass is wielded together with longer rods of greater masses, it will be perceived as shorter than it would when wielding shorter rods of lesser masses. Also, with previous experience of handling dense rods (with vision also provided), the scaling coefficients are adjusted to be smaller, resulting in underestimation of rod length, whereas, if a light rod has been handled, the scaling coefficients become larger, resulting in overestimation of rod length.

Similar findings were observed in the study of context effects (McKenna, 1984), where the judgment of an item is affected by the response of a neighboring item. It is displaced either in the same direction (assimilation) or in the opposite direction (contrast) of simultaneous or recently experienced values. Warren (1985) thought it was caused by the shift of the criteria used for evaluating stimuli, and that it was the contribution "of a general adaptive strategy that organizes and calibrates our perceptual interpretation of sensory input so as to ensure appropriate behavior" (p. 583). He also conceded that such adaptive strategy would lead to perceptual errors in special conditions.

Contrary to the above analysis, C. C. Pagano (personal communication, November 1994) suggested that, in holding rods stationary, muscle tremor might reveal the moment of inertia of the rod, which specifies rod length. Such arguments fit very nicely into the concept of a one-to-one mapping between i:variant patterns of physical stimulation and perception (Turvey, Solomon, \& Burton, 1989). It has been claimed that the specifications for orientation and length of a rod all come from a hyper number, the inertia tensor matrix, from which the eigenvalue and eigenvector can be derived. The eigenvalue specifies length, and the eigenvector specifies direction. In this view, the eigenvalue and the eigenvector are unique and are specific to rod length and direction. Perception using this in- formation is direct. No modification of the scaling coefficients by contextual experience is required. According to this view, the availability and the detection of a unique and specific invariant guarantees accurate perception of the environment. Indeed, there are many cases, such as the perception of time-to-contact in humans, animals, and insects (Lee, Lishman, \& Thomson, 1982; Lee \& Reddish, 1981; Turvey, Carello, \& Kim, 1990; Wager, 1982), which show that perception may be achieved through such direct detection of a unique and specific invariant. Yet, in haptic perception of rod length, this may not be the case.

Thus, we have identified two positions in the understanding of the haptic judgment of rod length. The first position argues that haptic judgment of length for unfamiliar rods does not give actual length (Chan, 1995). Rather, length perception depends on two kinds of situational factors. One is manipulation, and the other is the context effect (e.g., perception is dependent on stimulus range and prior experience). The former determines which parameters are used for perception. The latter affects the scaling coefficients for length judgments. The second position claims that haptic perception results in the detection of a unique and specific complex particular, the inertia tensor, for the specification of actual length. According to this view, adequate manipulation is necessary only to reveal the unique invariant for perception of a specific property, but manipulations do not alter the invariant to be detected. (Yet, the claim that inertia tensor can be detected even with the tremor of the hand while holding the rod stationary denies the need for adequate manipulation.) Any situational bias does not exist. Even if perception of rod length is not identical to the actual length, they are close.

The objective of this research was to determine which of the two positions better explained haptic perception of rod length. Two questions were addressed:

1. Will a unique and specific invariant be detected across different manipulations or will different parameters be used for length perception with different manipulations? The question can be asked differently. Does manipulation only reveal a unique and specific invariant or does manipulation determine the parameters or the combinations of parameters for specifying length?

2 . What, if any, are the context effects on the scaling coefficients in the haptic perception of rod length?

Three experiments were conducted to resolve these two questions.

\section{EXPERIMENT 1 \\ Effect of Manner of Manipulation on Perceived Length}

This experiment was undertaken to investigate whether different dynamic variables (weight, static torque, or moment of inertia) were responsible for different length specifications dependent on manner of manipulation. As shown in Figure 1, different force structures are produced in the wrist when a horizontal or vertical rod is being held or wielded. When a horizontal rod is held with the wrist fixed (Figure 1a), the mass of the rod produces a down- 

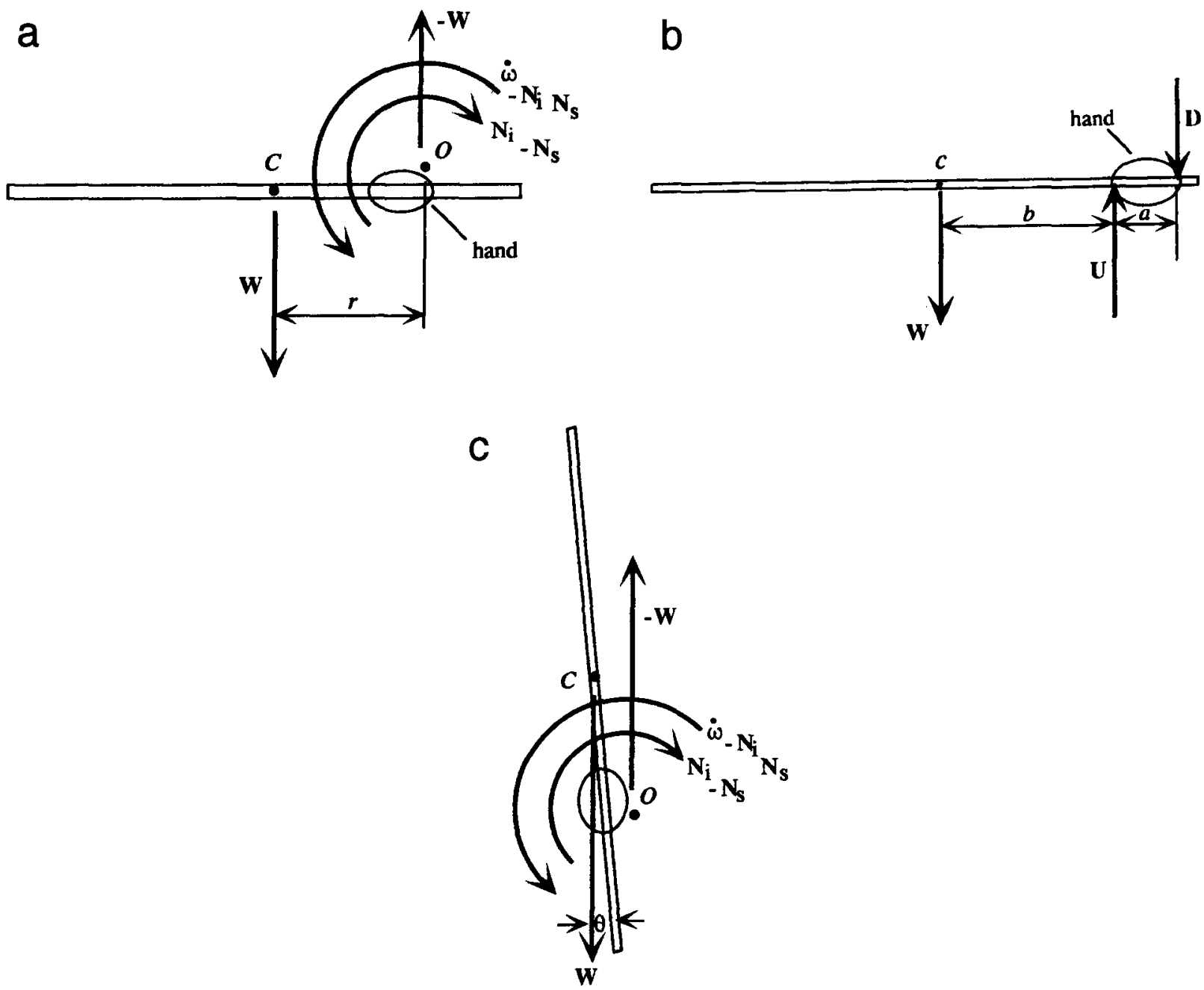

Figure 1. Free body diagrams showing force structure in different manipulations indicating the center of mass of the rod, $c$, and the wrist as the pivot point, $o$ : (a) holding and wielding a horizontal rod, (b) upward thrust and kick on a horizontal rod in holding it with $U=-W(a+b) / a$, (c) holding and wielding a vertical rod.

ward gravitational pull, $\mathbf{W}$, along the center of mass of the rod. Except when the center of mass is held, an anticlockwise static torque, $\mathbf{N}_{\mathrm{s}}$, is also produced on the wrist, pulling the left tip of the rod downward. To maintain the wrist stationary, an upward pull, $-\mathbf{W}$, and a counter torque, $-\mathbf{N}_{\mathrm{s}}$, have to be produced at the wrist. In such a condition, $\mathbf{N}_{\mathrm{s}}$ is sensitive to the position of the hand on the rod similar to the first moment of mass (the distribution of mass around an axis, hereafter called first moment), $\mathbf{M}(\mathbf{M}=m \mathbf{r}$, where $\mathbf{r}$ is the moment arm), which depicts the distribution of mass with reference to an axis (a vertical line through the wrist in this case). Actually, Hoisington (1920) found that perceived length of rods held at a horizontal position is dependent on mass distribution. Carello et al. (1992) also claimed that when a rod is held and not wielded, perceived reachable distance is also constrained by the first moment. In fact, it is the static torque which produces muscle strain that is used for length perception. Only when the rod is held horizon- tally does the static torque produced correspond to the first moment. Thus, it is appropriate to use the first moment to represent the rotational tendency at the wrist when the rod is held horizontally.

When the rod is wielded along the $y z$ plane (see Figure 4), an inertia torque, $\mathbf{N}_{i}$, is also produced. This inertia torque is a product of moment of inertia, $I$, a resistance to rotational acceleration called moment of inertia, and angular acceleration, $\dot{\omega}$ (see Appendix for details). The rotational torque covaries with the angular acceleration, and the moment of inertia is a property of the wielded rod which remains invariant. The inertia torque, $\mathbf{N}_{i}$, produced on the hand is always opposite to the direction of rotation. Thus, to maintain motion, a countertorque, $-\mathbf{N}_{i}$, has to be produced in the direction of $\dot{\omega}$.

A static torque can be represented by a couple (two opposite but noncollinear forces). Thus, for a horizontal rod held stationary, the effect of the static torque, $-\mathbf{N}_{\mathrm{s}}$, and the upward force, $-\mathbf{W}$, at the wrist (as shown in Fig- 
ure la) can be represented alternatively by two forces, $\mathbf{U}$ and $\mathbf{D}$ (as shown in Figure 1b). In fact, these two descriptions correspond to the force-torque model and weightpercept model proposed by Lederman, Ganeshan, and Ellis (in press). $\mathbf{U}$ is an upward thrust from the index finger reactive to the pressure of the rod, and $\mathbf{D}$ is a downward force from the heel of the hand reactive to the kick of the rod (Hoisington, 1920). Carello et al. (1992) showed that just $\mathbf{U}$ or $\mathbf{D}$ can be used for length perception. Lederman et al. (in press) claimed that participants judged rod length on the basis of a subjective "weight percept" which can be interpreted as the percept related to the upward thrust, $\mathbf{U}$.

For a vertical rod, similar force structures are produced (Figure 1c). When it is in a stationary condition, an upward thrust equal to the weight of the rod has to be produced. Because $\theta$ is small and $\sin \theta$ is negligible ( $\sin \theta$ affects static torque at the wrist), static torque experienced at the wrist is mainly dependent on the weight of the rod only. When the vertical rod is wielded, an additional counter inertial torque, $-\mathbf{N}_{\mathrm{i}}$, has to be produced.

Thus, logically, the length of a horizontally held rod can be specified only by weight, $\mathbf{W}$, and static torque, $\mathbf{N}_{\mathrm{s}}$ (represented by the first moment, $\mathbf{M}$, or the upward thrust, $\mathbf{U})$, and the length of a horizontally wielded rod can be affected by weight, $\mathbf{W}$, static torque, $\mathbf{N}_{\mathrm{s}}$, and moment of inertia, $I$. With vertical rods, length is mainly specified by weight, $\mathbf{W}$, when the rod is held stationary, and by moment of inertia, $I$, and weight, $\mathbf{W}$, when it is wielded. The above hypotheses can be checked by comparing four ways of manipulation: holding a horizontal rod, wielding a hor- izontal rod, holding a vertical rod, and wielding a vertical rod.

When rods are held at any fixed position (e.g., at one end or at $1 / 3$ of its length), the mass, the static torque, and the moment of inertia of the rod increase together with the increase of actual length (or with added mass). It is quite impossible to dissociate the effect of static torque from the effect of moment of inertia on length perception. This has been the case for most of the experiments done heretofore. In this experiment, various rod lengths $(50-100 \mathrm{~cm})$ were grasped, with the length forward of the hand kept constant. The $50-\mathrm{cm}$ rod was held close at one end, whereas the longest three rods were held roughly at the middle. With such an arrangement, as shown in Figure 2, when rods are held horizontally, with the increase of actual rod length, first moment decreases, whereas both the moment of inertia and weight increase. Thus, in this experiment, with the increase of actual length, perceived length that is dependent on first moment would decrease, whereas perceived length that is dependent on weight or moment of inertia would increase. It is expected that, with the above dissociation between static torque, moment of inertia, and weight, the effect of manipulation in determining the variables to be used in length perception can thus be demonstrated.

\section{Method}

Subjects. Thirty-two undergraduates at The Chinese University of Hong Kong were paid to participate in four different manipulation conditions. All of them indicated they were right-handed. Eight (4 male and 4 female) were assigned to hold horizontal rods, 8 (4



Figure 2. The dissociation between static torque, moment of inertia, and weight with the increase of actual rod length. The first moment (or static torque experienced at the wrist when the rod is horizontal) and upward thrust are represented as alternatives for detecting static torque. 
male and 4 female) to hold vertical rods, 8 ( 3 male and 5 female) to wield horizontal rods, and 8 ( 3 male and 5 female) to wield vertical rods.

Apparatus. Six homogeneous aluminum rods (1-cm diam) 50 , $60,70,80,90$, and $100 \mathrm{~cm}$ long and with masses of $103,122.6$, $143.4,164.6,183.45$, and $203 \mathrm{~g}$, respectively, were used. To occlude the hand and the grasped rods, a 3-m-wide and 2-m-high curtain hung from $2.1 \mathrm{~m}$ above the floor. A 0.3-m-long slit, with its lower end $0.73 \mathrm{~m}$ above the floor, was provided for the subjects to insert their right hands through the curtain (Figure 3). A chair back, $0.8 \mathrm{~m}$ high, was placed below the slit as an armrest. For reporting length, a "length measurer," $1.85 \mathrm{~m}$ long and $0.93 \mathrm{~m}$ high, was used. The length measurer had a sliding marker connected with a string mounted on a board. By pulling the string up or down at the starting edge of the board, the marker could be moved along a meter rule fixed by the side of the board hidden from the participants. The perceived length of a rod could then be translated to the distance of the marker from the starting edge. The length measurer was placed along the occluding curtain in front of the participant.

Procedure and Design. A subject positioned his/her right arm through the slit in the curtain and rested the forearm on the armrest behind the curtain. Thus, in all four conditions, the elbow was stationary and the wrist could be considered as fixed and acting as a pivot. Rods of different lengths were placed by the experimenter horizontally or vertically into the subject's right hand; these were always gripped by the subject with the wrist at about $49 \mathrm{~cm}$ from the front end and about $6 \mathrm{~cm}$ from the rod axis (Figure 4). The subject gripped the rods firmly with the thumb positioned adjacent to the index finger. This step was taken to ensure that the subjects could not move the rod with the thumb independently of the wrist so that the wrist would act as the pivot point of the rod-hand system.

The subjects were assigned randomly to one of the four conditions of manipulation. In the two holding conditions, they were cautioned to keep the rod stationary. For the two wielding conditions, they swung the rods, with the wrist as the pivot, slightly up and down or forward and backward as the case might be. For horizontal rods, the subjects pointed the rod forward parallel to the curtain (as shown in Figure 4). For vertical rods, the front end was pointed upwards by rotating the wrist $90^{\circ}$ from the horizontal position. In each trial, after perceiving the length of the rod, the subjects moved the marker of the length measurer away from themselves with the left hand to indicate the perceived length of the whole rod held or wielded in the right hand. The subjects were encouraged to take as much time as necessary in order to perceive the rod length as accurately as possible. At the end of each trial, the rod was taken away and the subjects moved the marker back to the starting edge. Before the experimental trials, the subjects were given a practice trial with a rod of similar material that was not used in the experiment.

In the experiment, the six rods were presented randomly in a block of trials. There were four blocks making for a total of 24 trials. Perceived length of the whole rod was measured in each trial.

The experiment was a $4 \times 6$ (manipulation $\times$ rod length) mixedsubjects design. It was expected that perceived rod length would depend on the variable detected in a specific manipulation, not necessarily proportional to the actual length.

\section{Results and Discussion}

The actual length, mass, moment of inertia, static torque as represented by first moment and the upward thrust, and the perceived lengths are listed in Table 1. An analysis of variance (ANOVA) showed that the interaction between length and manipulation was significant $[F(15,140)=$ $9.98, p<.0001]$, indicating that the effect of actual rod length on perceived length was dependent on manipulation (Figure 5). Nested analysis of ANOVA showed that interaction between length and manipulation was significant for holding and wielding horizontal rods $[F(5,70)=$ $13.06, p<.0001]$, for holding horizontal and vertical rods $[F(5,70)=19.37, p<.0001]$, for holding horizontal rods and wielding vertical rods $[F(5,70)=22.84, p<.0001]$, and for wielding horizontal and vertical rods $[F(5,70)=$ $2.87, p<.03]$. All these interactions result from a dissociation of static torque with weight or with moment of inertia (see Figure 2). As shown in Figure 5, perceived rod length is clearly a function of manipulation. When compared with Figure 2, it is not difficult to match the variable exploited with the means of manipulation. As indicated in the results, direct perception cannot be claimed for haptic perception of rod length.
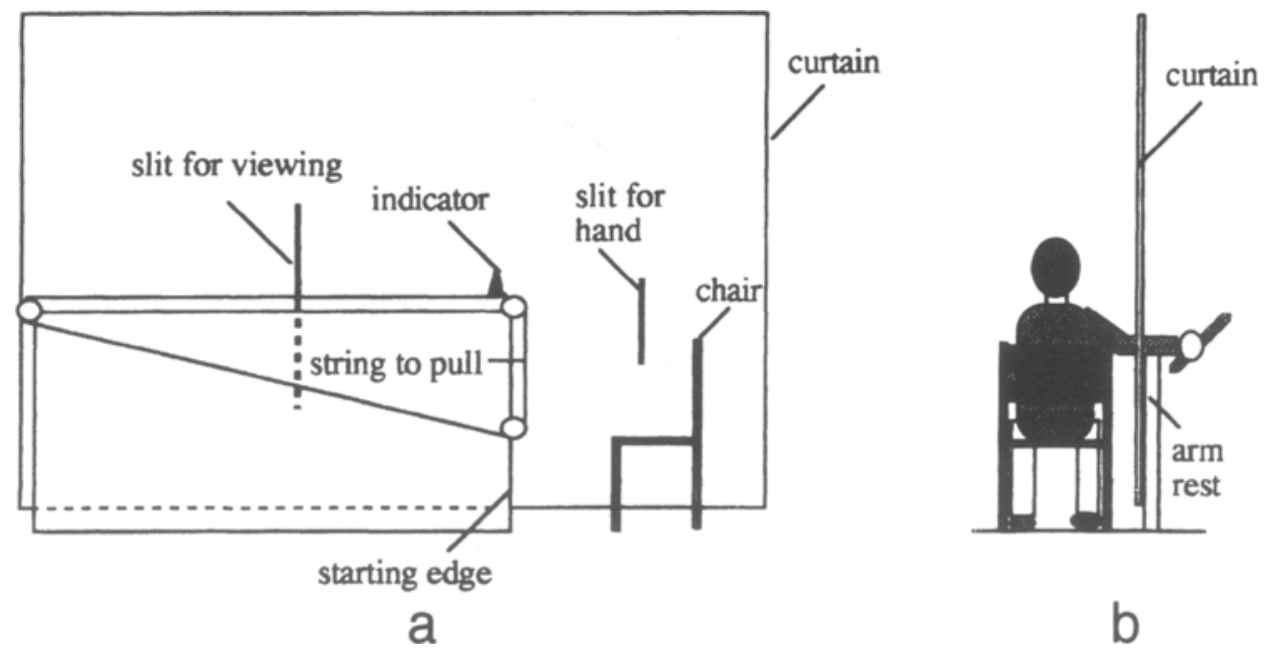

Figure 3. Apparatus. (a) Arrangement of the apparatus with the length measurer placed in front of a participant along the curtain. The experimenter can read the scale by looking through another slit in the curtain. (b) Side view, with a participant wielding a rod by the inserted arm on the other side of the curtain. 


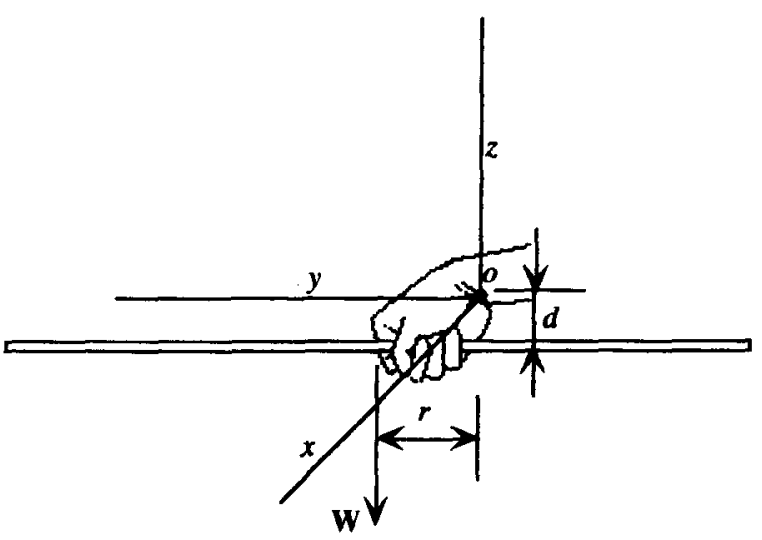

Figure 4. A horizontal hand-held rod with respect to the coordinate system, showing also the location of the grip and the pivoted point. The rod is grasped between its two ends pivoted at the wrist. The weight $(W)$ acts downward through the center of mass. The wrist was kept $49 \mathrm{~cm}$ from the tip, and $d$ is about $6 \mathrm{~cm}$. For vertical rods, the wrist rotates $90^{\circ}$ with the tip of the rod pointing upward.

Regression analysis was used to show which dynamic was exploited under which manipulation. Perceived rod length of each of the manipulations was regressed on the weight of the rods, moment of inertia, first moment, and the upward thrust at one edge of the hand in log log plots. As shown in Figure 6, weight of rod predicts perceived length the best when rods were held vertical $\left(R^{2}=\right.$ .98 ), showing that weight was the detected variable in such conditions. It predicted perceived length moderately well when rods were wielded $\left(R^{2}=.89\right.$ for vertical rods and .80 for horizontal rods), because moment of inertia, exploited in wielding conditions, and weight are both functions of rod mass. It predicted perceived length most poorly when the rods were held horizontally $\left(R^{2}=.34\right)$. Figure 7 shows regression of perceived lengths on moment of inertia, $I_{\mathrm{xx}}$ about the major axis of rotation, $x .^{3}$

The results indicated that moment of inertia predicts perceived length the best when rods are wielded $\left(R^{2}=\right.$ .98 for vertical rods and $R^{2}=.92$ for horizontal rods), showing that moment of inertia is used for length perception mainly when rods are wielded. It predicts perceived length moderately well when the rods are held vertically $\left(R^{2}=.84\right)$ because weight correlates with moment of inertia. It predicts perceived length the most poorly with rods held horizontally $\left(R^{2}=.07\right)$. As shown in Figure 8a, first moment does not seem to be exploited for perceived length in any of the manipulations. It has surprisingly poor prediction even when the rods were held horizontally $\left(R^{2}=.114\right)$. Although it predicts perceived length very well with the other three conditions, the fact that the regression coefficients are negative suggests that, in all three conditions, perceived length is proportional to parameters (such as weight and moment of inertia) that are negatively correlated to the first moment (Figure 2). When regressed on the upward thrust from the edge of the hand (Figure $8 \mathrm{~b}$ ), perceived length was predicted reasonably well when rods were held horizontally $\left(R^{2}=.82\right)$ and very poorly in all the other conditions $\left(R^{2}<.3\right)$. Results in regressional analysis, as shown in Figures $8 \mathrm{a}$ and $8 \mathrm{~b}$, suggest that static torque in the wrist is not used for judgment of rod length when the rods are held horizontally; upward thrust from the edge of the hand is used instead (see note 1). It is certainly more economical to attend to the upward thrust, which gives combined information about the magnitude of static torque and the weight of the rod.

The simple regression shows to what extent a physical parameter can explain the variance in perceived length under each manner of manipulation. With respect to Stevens's power law, $\psi(\lambda)=b x^{p}$, the slope of each regression line gives the exponential coefficient, $p$, of the power equation that scales a physical parameter with magnitude $x$ to perceived length, $\psi(\lambda)$. Because of the high intercorrelation between the physical parameters, multiple regression cannot be used.

In sum, the results show clearly that the manner of rod manipulation determines the parameters exploited in the perception of rod length.

\section{EXPERIMENT 2}

\section{The Effect of Range on the Scaling Coefficients}

In the study of Solomon and Turvey (1988), with participants wielding solid aluminum rods, it was found that long rods were usually underestimated while short rods were overestimated. In other words, the power function, which maps the detected physical parameter such as weight, static torque, or moment of inertia onto perceived length, changes its scaling coefficients with the magnitude of the parameter in each trial. Solomon and Turvey's

Table 1

Mean Perceived Lengths (in Centimeters) With Four Methods of Manipulation in Experiment 1

\begin{tabular}{|c|c|c|c|c|c|c|c|c|c|c|c|c|}
\hline \multirow{4}{*}{$\begin{array}{l}\text { Rod } \\
\text { Length } \\
\text { (cm) }\end{array}$} & \multirow{4}{*}{$\begin{array}{l}\text { Rod } \\
\text { Weight } \\
\text { (gwt) }\end{array}$} & \multirow{4}{*}{$\begin{array}{c}\text { Moment } \\
\text { of Inertia } \\
I_{x x} \\
\left(\mathrm{~g} \cdot \mathrm{cm}^{2} / 1,000\right)\end{array}$} & & & \multicolumn{8}{|c|}{ Perceived Length } \\
\hline & & & \multicolumn{2}{|c|}{ Rotational Tendency } & \multirow{2}{*}{\multicolumn{2}{|c|}{$\begin{array}{l}\text { Holding } \\
\text { Horizontal }\end{array}$}} & \multirow{2}{*}{\multicolumn{2}{|c|}{$\begin{array}{l}\text { Holding } \\
\text { Vertical }\end{array}$}} & \multirow{2}{*}{\multicolumn{2}{|c|}{$\begin{array}{l}\text { Wielding } \\
\text { Horizontal }\end{array}$}} & \multirow{2}{*}{\multicolumn{2}{|c|}{$\begin{array}{l}\text { Wielding } \\
\text { Vertical }\end{array}$}} \\
\hline & & & First Moment & Thrust & & & & & & & & \\
\hline & & & $(\mathrm{g} \cdot \mathrm{cm})$ & (gwt) & $M$ & $S D$ & $M$ & $S D$ & $M$ & $S D$ & $M$ & $S D$ \\
\hline 50 & 103.00 & 79.66 & $2,369.00$ & 296.13 & 81.36 & 18.4 & 50.61 & 11.8 & 73.63 & 22.1 & 72.66 & 17.1 \\
\hline 60 & 122.60 & 80.92 & $2,206.80$ & 275.85 & 73.61 & 13.8 & 55.37 & 10.9 & 74.47 & 16.0 & 74.50 & 18.1 \\
\hline 70 & 143.40 & 87.96 & $1,864.20$ & 233.03 & 71.69 & 11.4 & 59.22 & 12.3 & 73.13 & 17.9 & 78.19 & 20.9 \\
\hline 80 & 164.60 & 104.26 & $1,316.80$ & 164.60 & 62.05 & 8.8 & 64.93 & 15.2 & 81.25 & 21.2 & 85.50 & 25.7 \\
\hline 90 & 183.45 & 132.09 & 550.35 & 114.66 & 62.94 & 10.4 & 65.79 & 18.5 & 83.78 & 20.6 & 92.58 & 26.2 \\
\hline 100 & 203.00 & 177.30 & 406.00 & 253.75 & 75.55 & 17.4 & 72.18 & 18.8 & 88.06 & 20.6 & 102.00 & 28.0 \\
\hline
\end{tabular}




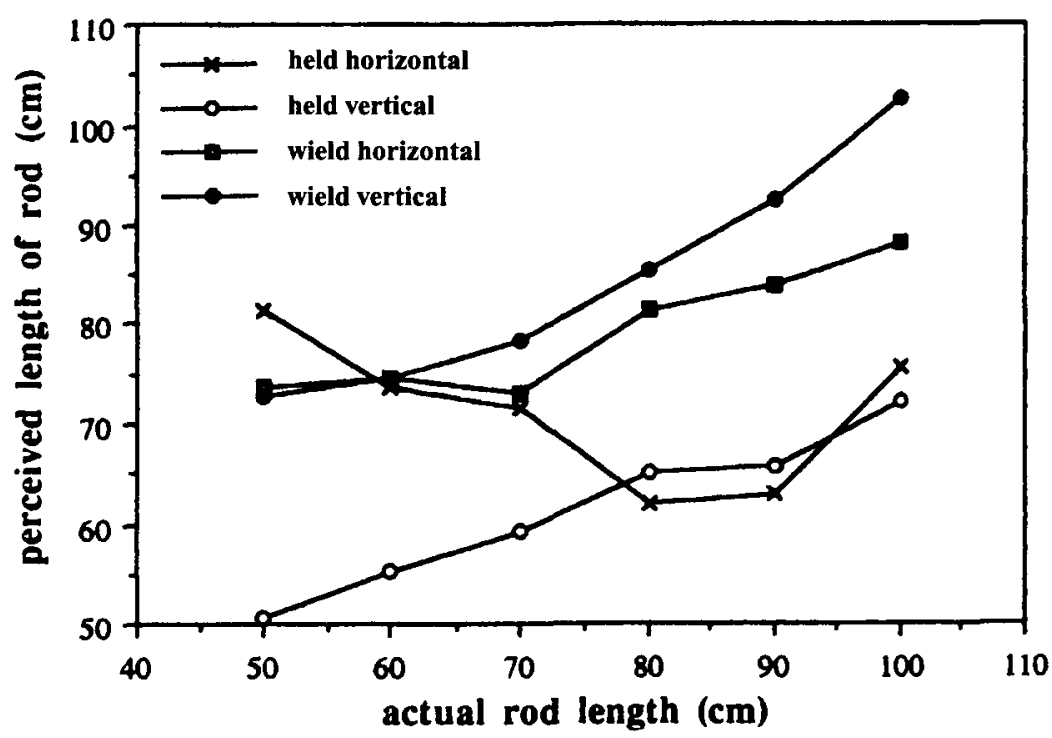

Figure 5. The effect of actual length on perceived length is dependent on the manner of manipulation (Experiment 1).

results suggest that the scaling coefficients are larger with shorter rods and smaller with longer rods. In a sense, this alteration of the scaling coefficients is adaptive, as argued above, because denser rods need smaller scaling coefficients while less dense rods need larger ones in order to bring perceived length closer to the actual length. Unfortunately, such a scaling alteration also causes errors in the judgment of length for unfamiliar rods. This experiment is designed to show that such change of the scaling coefficients does occur and is affected by the range of rods to be judged successively in a set.

A rod was wielded with a number of shorter rods for judgment of rod length. Then the same rod was wielded with a number of longer rods for judgment of rod length. If the scaling coefficients change with the range of rods wielded in a set, as predicted above, the perceived length of the rod should be longer when wielded with shorter rods than when wielded with longer rods.

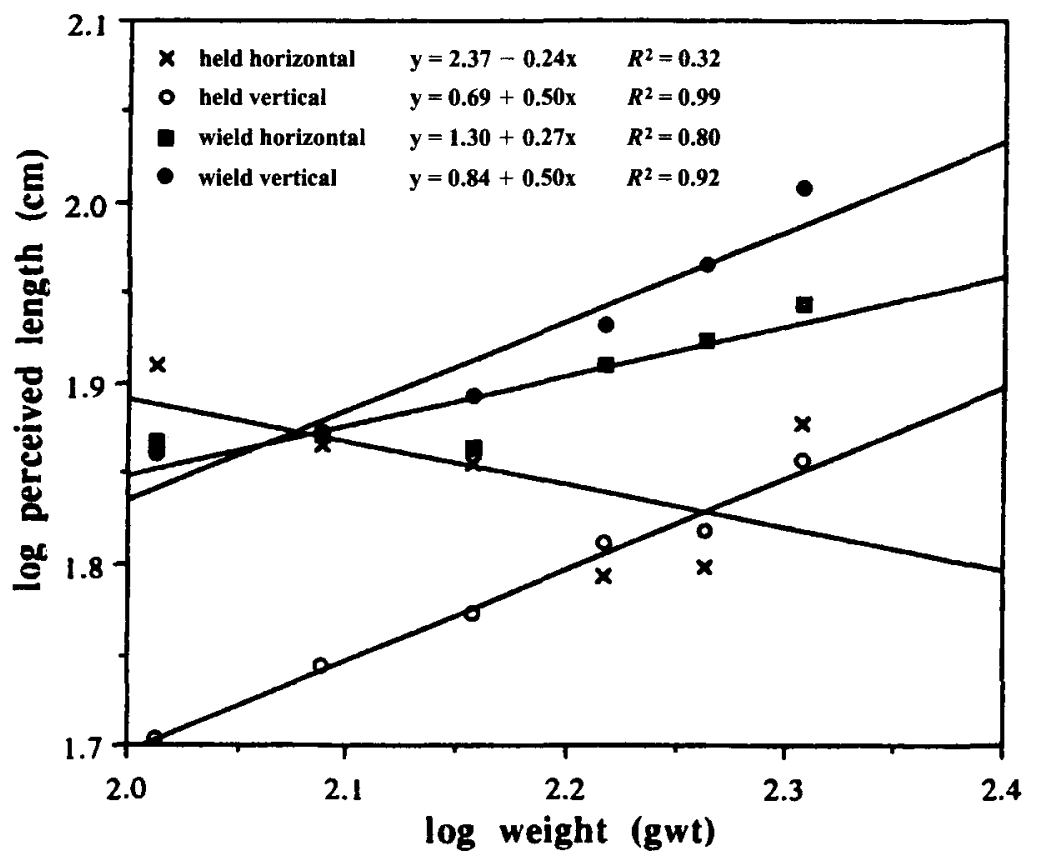

Figure 6. Regression of perceived lengths in four different manipulations on weight of the rod, in a $\log \log$ plot (Experiment 1). 




Figure 7. Regression of perceived lengths in four different manipulations on moment of inertia in a $\log \log$ plot (Experiment 1).

\section{Method}

Subjects. Eight undergraduates in an introductory psychology course at The Chinese University of Hong Kong participated in partial fulfillment of a course requirement. All of them indicated they were right-handed. None of these subjects had participated in Experiment 1.

Apparatus. Seven homogeneous aluminum rods $(1 \mathrm{~cm}$ diam), $40,50,60,70,80,99$, and $110 \mathrm{~cm}$ long with masses of $82.7,103$, $122.6,143.4,164.6,201.2$, and $224.3 \mathrm{~g}$, respectively, were used. The first set included the first four rods; the second set included the last four. The $70-\mathrm{cm}$ rod was the longest rod in the first set but the shortest in the second set. The curtain and the length measurer were the same as in Experiment 1.

Procedure and Design. As in Experiment 1, a subject positioned his/her right arm through the slit in the curtain to grip and wield the rods, and produced judgments of length with the length measurer. In this experiment, all rods were presented horizontally and were wielded at one end with the grip position at $5 \mathrm{~cm}$ from the rear end. Each set of four rods was presented to a subject twice in a block of trials in random order. There were four blocks, making for a total of 32 trials. The order of presentation of the two sets of rods was counterbalanced by using the ABBA (alternated with BAAB between subjects) format for each subject. Perceived length on each trial was measured. Before the experimental trials, the subjects were given a practice trial with a rod that was not used in the experiment.

The experiment was a $2 \times 4$ (range $\times$ rod length) within-subjects design. It was expected that, although longer rods are perceived as being longer, perceived length of the same rod would be shorter when it was wielded with the longer set of rods than when it was wielded with the shorter set of rods.

\section{Results and Discussion}

The mean perceived lengths of the shorter set of rods $(40,50,60$, and $70 \mathrm{~cm})$ are $41.19,54.91,66.28$, and $79.53 \mathrm{~cm}$ with standard deviations of $5.92,6.02,8.10$, and $8.43 \mathrm{~cm}$, respectively. Those of the longer set of rods $(70,80,99$, and $110 \mathrm{~cm})$ are $73.28,84.22,115.44$, and
$118.19 \mathrm{~cm}$ with standard deviations of $5.35,9.72,36.01$, and $15.10 \mathrm{~cm}$, respectively. An ANOVA showed that the effect of range was significant $[F(1,7)=92.97, p<.0002]$, indicating that, between different sessions, longer rods were perceived as being longer. The effect of length was significant $[F(3,21)=24.18, p<.0001]$, indicating that within a session, longer rods were perceived as being longer. Although perceived lengths are generally quite accurate vis-à-vis actual lengths, perceived length of the $70-\mathrm{cm}$ rod was longer when judged with shorter rods $(79.53 \mathrm{~cm})$ than when judged with longer rods $(73.28)$. A $t$ test showed that the difference between these two measures was significant $[t(7)=2.54, p<.04]$.

The results show clearly that, although a definite physical variable is detected in the perceptual process during a certain manipulation, the function that maps the detected variable onto perceived length varies according to situation. Thus, when horizontal rods are wielded, perceived length is mainly a function of static torque, $\mathbf{N}_{\mathrm{s}}$, and of moment of inertia, $I$, so that perceived length equals $f\left(\mathbf{N}_{\mathrm{s}}, I\right)$. The function, $f$, may change due to context effects. More specifically, the change can be thought of as an alteration of a certain constant term. With perceived length represented by Stevens's power law, perceived length equals $b \mathbf{N}_{\mathrm{s}}^{p} I^{q}$, where $b, p$, and $q$ are constants. It is the change of these constants in response to contextual changes that alters the perceived length.

Results showed a context effect in the haptic perception of rod length. Hollingworth (1928) argued that after repeated exposure to stimuli of different values, the evaluation of individual items tends toward a point of neutrality such that stimuli are reported with less extreme values. This concept of neutrality quite accurately de- 

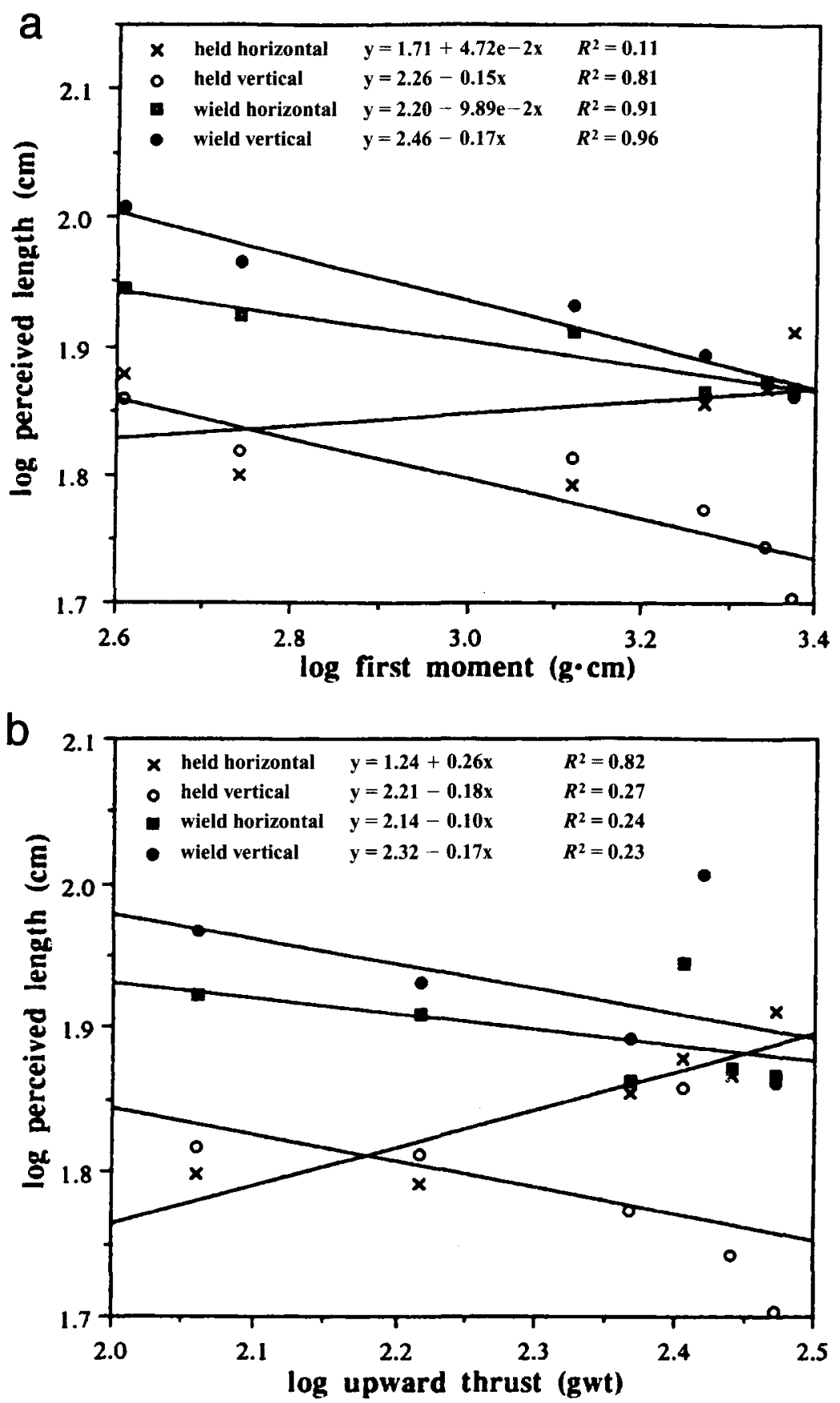

Figure 8. Regression of perceived length in four different manipulations on static torque in a $\log \log$ plot (Experiment 1): (a) Static torque experienced at the wrist is represented by the first moment of mass; (b) static torque is represented by the upward thrust from the edge of the hand on the rod.

scribes the results of Solomon and Turvey's (1988) study, where longer rods were underestimated while shorter rods were overestimated. Yet, results from Experiment 2 do not agree with this explanation. At the extreme measures $(40$ and $110 \mathrm{~cm})$, actual lengths were not different from perceived lengths. The length of the $40-\mathrm{cm}$ rod was reported as being $41.18 \mathrm{~cm}(t<1)$ and that of the 110 $\mathrm{cm}$ rod was reported as $118 \mathrm{~cm}(t<1)$. In fact, for both sets of rods, the range of perceived length was greater than that of the actual length. For the short set, the actual range was $30 \mathrm{~cm}$, while the perceived range was $38.34 \mathrm{~cm}$. For the long set, the actual range was $40 \mathrm{~cm}$, while the perceived range was $44.91 \mathrm{~cm}$. This may be caused by the splitting of the whole range of rods into two different sets of narrower range, with each set evaluated in one block of trials. When rods are similar in actual lengths and their 
relative lengths can be discriminated easily, perceived lengths tend to diverge rather than converge to a point of neutrality. For this reason, the $70-\mathrm{cm}$ rod was perceived as being longer in the short set than in the long set.

Results in this experiment can be compared with a contrast effect in which a stimulus would be perceived in the opposite direction as an adapting stimulus. Thus, after repeated exposure to a curve, a straight line would be perceived as a curve bending in the opposite direction (Gibson, 1933); after repeated exposure to downward moving bars, stationary bars would appear to move upward (waterfall illusion). In this experiment, when measured with short rods, a rod appeared to be longer than when it was measured with long rods.

\section{EXPERIMENT 3 The Effect of Prior Experience on the Scaling Coefficients}

In Solomon and Turvey's (1988) study, in which participants wielded solid aluminum rods, although perceived length across participants was found to be close to actual length, individual participants differed greatly. When perceived length was regressed on actual length, the regression coefficients ranged from 0.574 to 0.911 among participants. Similar variation was also reported in Chan and Turvey (1991). It was hypothesized that such individual differences in length judgments were due to the effect of prior haptic experience, which is different among different individuals. The question examined here was: Does prior experience in handling rods of a given density affect length judgment? It was hypothesized that it would.

It is likely that such effect of prior experience produces not a change in sensory discriminability but a change in judgment. It might be that, as demonstrated by the method of magnitude estimation, in judging the length of a rod, a certain standard has to be used for comparison. Individual differences may be explained by the differences in the standard used by individuals. Whether the effect of prior experience is a sensory change or a judgment change, it must be effected through an adjustment in the scaling coefficients. Such adjustment in scaling coefficients, as argued above, is adaptive, because perceived length depends on the detection of physical parameters that have no unique or specific relationship to the actual rod length. Thus, the scaling coefficients have to be adjusted according to actual length.

To test the hypothesis that prior experience affects perceived length, participants were asked to judge rod lengths after experiencing handling either a dense rod or a light rod with visual feedback. It was expected that the lengths of rods judged after the dense rod had been handled would be shorter than those judged after the light rod had been handled. In order to examine the interaction between the effect of prior experience and range set on perceived length, both range set and prior experience were included as independent variables.

\section{Method}

Subjects. Nine undergraduates in an introductory psychology course at The Chinese University of Hong Kong participated in partial fulfillment of a course requirement. All of them were identified as being right-handed. None of them had participated in Experiment 1 or Experiment 2.

Apparatus. The apparatus was the same as that used in Experiment 2 except that two $60-\mathrm{cm}$ rods ( $1-\mathrm{cm}$ diam) were used for practice by the subjects before they made their length judgments. Of the two practice rods, one was made of copper (dense) and the other was made of hollow steel (light).

Procedure and Design. The procedure was the same as in Experiment 2 except that both sets of rods were presented in successive blocks of trials after a subject had handled either a heavy or a light rod. At each level of prior experience, the subject began by judging, once, the length of a practice rod (dense or light) with the length measurer. Then the practice rod was handed to the subject so that he/she could find out whether its length had been overestimated or underestimated. Then the subject used the practice rod to touch each of four different targets on a wall four times. The rationale for this procedure was that pointing to the targets would provide visual and haptic information about the length that would affect the scaling coefficients. The order of presentation of the two levels of prior experience was counterbalanced among the subjects. Within each level, both sets of four rods were presented twice randomly with each set in one block. The subjects evaluated rod length in each of the 32 trials.

The experiment was a $2 \times 2$ (experience $\times$ range) within-subjects design. It was expected that the perceived length of the rod would be shorter when wielded after the subject had handled the denser rod than after he/she had handled the less dense rod and would be shorter when wielded in the longer than when wielded in the shorter set.

\section{Results and Discussion}

Prior experience, range set, and actual rod lengths are listed in Table 2 with their mean perceived lengths and standard deviations. An ANOVA showed that the effect of prior experience was significant $[F(1,8)=18.84, p<$ $.003]$. Given Stevens's power law for the scaling relationship between perceived value and stimulus intensity, the effect of prior experience can only mean that the scaling coefficients were affected by prior experience. The effect of range set is significant $[F(1,8)=65.45, p<.0001]$, as is the interaction between prior experience and range set $[F(1,8)=12.7, p<.008]$ (Figure 9). The significance of range set is trivial because the longer set had greater value of moment of inertia, which is the stimulus intensity $x$ in the power of $\psi(\lambda)=b x^{p}$. The significance of the interaction between prior experience and range set is nontrivial and can most appropriately be explained as the result of the increase of $b$ in the power law.

An ANOVA on the perceived length of the $70-\mathrm{cm}$ rod showed that the effect of prior experience was significant $[F(1,8)=11.3, p<.02]$ but that the effect of range set was not $[F(1,8)=3.927, p>.05]$. In comparison with the results from Experiment 2, this indicates that the effect of range set is weak and would become insignificant if other factors were involved in an experiment.

Results show that prior experience in handling the practice rod with visual feedback recalibrates the length criterion in the haptic system. The criterion shifts after the rods have been handled even if only a few times. The re- 
Table 2

Mean Perceived Lengths (in Centimeters) With Two Context Effects in Experiment 3

\begin{tabular}{lcrr}
\hline & Rod Length & \multicolumn{2}{c}{ Perceived Length } \\
\cline { 2 - 4 } Range Set & (cm) & $M$ & $S D$ \\
\hline \multirow{4}{*}{ Short } & Prior Experience: Light & \\
& 40 & 46.61 & 12.68 \\
& 50 & 58.81 & 12.92 \\
Long & 60 & 69.72 & 7.31 \\
& 70 & 86.06 & 16.18 \\
& 70 & 79.56 & 11.03 \\
& 80 & 94.36 & 15.78 \\
& 99 & 11.64 & 20.85 \\
Short & 110 & 122.17 & 22.41 \\
& Prior Experience: Dense & \\
& 40 & 45.42 & 10.39 \\
& 50 & 56.17 & 7.93 \\
Long & 60 & 67.00 & 13.74 \\
& 70 & 73.94 & 11.57 \\
& 70 & 69.06 & 12.58 \\
& 80 & 79.03 & 15.39 \\
& 99 & 92.17 & 18.77 \\
& 110 & 96.89 & 22.36 \\
\hline
\end{tabular}

sults also suggest that the criterion affects perceptual judgment by altering the scaling coefficients in the power law. Such modification helps in the perception of the actual length of a rod even though there is no detectable haptic parameter that maps isomorphically onto the actual rod length.

\section{GENERAL DISCUSSION}

Since Helmholtz's proposal of unconscious inference, perception has been understood to be the result of the integration of percepts and concepts. Perception of the ex- ternal world has to be mediated by what we know about the world. The ecological approach, however, argues that if perception is mediated, there would be no sound epistemological support for perception. Perception would depend on knowledge, which in turn would depend on perception. The argument would be circular. Perception would be similar to guessing. Starting with J. J. Gibson, ecological psychologists have argued that perceptual knowledge must be primary. Gibson $(1966,1979)$ showed that, in both the haptic and visual modalities, information is so rich that perception of the external world can be direct without mediation. Knowledge may be necessary if perception starts with the integration of elementary sensations that underrepresent objects and events in the external world. If perception starts with the detection of information that is rich, then mediation is not necessary. The information that gets the job done is claimed to be the stimulation pattern that remains unchanged through the flux of changing sensations. This unchanging pattern is called an invariant. It is asserted that information is specific to the environment and perception is specific to information. Detected information therefore specifies the external world accurately. A stronger position of such information-perception specificity even argues that there is a one-to-one mapping between information and perception: a certain perception has to be specified lawfully by a unique and specific invariant out in the environment. Learning involves only the education of the attentional system to detect the specific invariant.

Such an argument of information-perception specificity was claimed in the area of haptic perception. It was argued that the length of a homogeneous rod could be specified accurately by the principal moments of inertia (Fitzpatrick et al., 1994; Solomon \& Turvey, 1988; Solomon, Turvey, \& Burton, 1989; Turvey et al., 1989), prop-



Figure 9. The effect of experience and set in the judgment of rod length (Experiment 3). 
erties of rods depending on mass distribution, which produce resistance to rotational acceleration in wielding. Yet there are logical, as well as empirical, anomalies against such an argument.

Information-perception specificity may hold only when there is, indeed, relevant information specifying a certain property of an object or event. However, there is no specific haptic information specifying the actual length of a rod. Chan (1995) showed that, in wielding rods of different densities, subjects perceived denser rods to be longer. Subjects appeared to be incapable of picking up any information for the accurate specification of the actual rod length, not even close to it. Moreover, in the present research, parameters other than moment of inertia are found to be involved in determining perceived length. Weight and static torque are even more important in the judgment of rod length when the rod is held stationary. Unlike Solomon and Turvey's (1988) study, where perceived length was found to be only a function of mass distribution, the present research shows that the manner of manipulation determines which parameter is more important in judging rod length. Also, Lederman et al. (in press) showed that both weight and torque are used in length perception, showing that moment of inertia is not the only information needed for length judgment.

When the moment of inertia is used to specify perceived length, the scaling function that maps moment of inertia onto perceived length depends on a number of factors. Chan (1995) showed that the diameter of the handle and even knowledge of rod diameter affect judgment of rod length independently of the actual length of the rod. The results of the present research show that the range of rod length presented and prior haptic experience in handling rods also affect perceived length independently of actual length.

Because of inaccuracy in the perception of unfamiliar rods, as shown above, the notion of lawful specification in haptic perception of rod length needs modifications. It appears that there is no one-to-one mapping between a unique and specific invariant and the actual length. Yet, on the other hand, athletes appear to be able to accurately perceive the lengths of unhomogeneous rods. They can, for example, accurately hit a ball with a familiar rod without looking at the rod. The mechanism for their accuracy appears to lie outside the lawful specification of rod length. Three issues are discussed below.

What is the role of manipulation in haptic perception of rod length? It is certainly true that, in perception, both "the environment and movement properties" are causally responsible for "the structured energy distribution available to a perceptual system" (Turvey et al., 1989, p. 387). The nature of manipulation determines the movement property inducing the energy distribution. Yet, manipulation has so far been treated as an active process revealing the unique invariant to be mapped to the perceived property. In this research, manipulation has been found to determine which of such dynamic parameters as weight, first moment, and moment of inertia is used to specify rod length. To perceive rod length accurately, people must therefore manipulate the tools in such manner that a parameter that is most sensitive to length is exploited. Moment of inertia, for example, which is proportional to length squared, is more sensitive to length than is weight, which is proportional to length only. Moreover, the manner of manipulation has to be constant such that the same parameter is used throughout the judgment.

How can the actual length be perceived with the haptic modality? The sensitivity to a physical property is very often modality specific: vision is sensitive to color and skin is sensitive to temperature, but not vice versa. If actual rod length can be perceived with the haptic modality, the available haptic information must have an accurate relationship with the actual rod length. Yet, not one of the available physical parameters, such as weight, static torque, and moment of inertia, which are sensitive to the haptic system, have an independent relationship with actual length. If perceived rod length were proportional to the magnitude of any one of these parameters, our haptic system could discern only the order of lengths among similar rods, but not the absolute length of a rod. If so, the perceptual accuracy of rod length when people are using tools such as a racket or a hammer cannot be explained.

In this research, the scaling coefficients that map detected physical parameters onto perceived lengths are found to be affected by contextual effects. This alteration of the scaling coefficients is nontrivial in haptic perception of rod length. With the practice of using a racket or a hammer, the scaling coefficients can be altered such that the perceived length can be made close to the actual length. In such a case, the accuracy of haptic perception of rod length depends on the adaptability of the haptic system in changing the scaling coefficients.

Implication for realism. Perception as a result of the detection of a unique and specific invariant, as proposed by the ecological approach, is a guarantee for realism: people can perceive the world as it is. In so doing, in each case of perception, there must be a unique and specific psychophysical function that maps a unique and specific invariant onto a perceived property. With such one-toone mapping, perception can be said to be direct without cognitive mediation. Such a position can be called absolute realism. Although direct perception may occur in the perception of time-to-contact and in the perception of the nature of a collision (Turvey \& Carello, 1986), but that is not the case in the haptic perception of rod length. There is no specific invariant or specific function in the haptic perception of rod length. There are, however, a number of physical parameters, and the scaling coefficients can be altered. Because different parameters can be mapped onto the perceived length, the mapping is many-to-one. For a specific parameter, because the scaling coefficients vary according to context effects, the mapping is one-to-many. In this case, absolute realism can never be realized. But, if practice can fix the values of the scaling terms for a specific rod with a specific manner of manipulation, the actual length can be perceived. In such a case, realism is achieved, not by having unique and specific invariant and function across different rods 
and manipulations, but by arbitrarily fitting the scaling coefficients through learning, so that mapping becomes unique and specific in each case. Perception is accurate only if the scaling terms can be fitted appropriately in learning. Thus, we can call such realism relative. Relative realism provides better adaptability than does absolute realism for systems that vary a lot between conditions. This is true for the handling of rods that have different densities, diameters, and shape. As mentioned by Cutting (1993), invariants may be rare in perception. For perceptions with systems that vary between conditions when there is no one-to-one mapping relationship, nature provides us with mechanisms that adjust for individual condition through learning.

In summary, I agree with the ecological approach; that is, if perception is mediated freely by mental processing, perception would be similar to guessing. But if mediation can be taken as the selection of parameters and the alteration of scaling coefficients, realism still can be guaranteed. In fact, for conditions in which there is no oneto-one mapping between invariants and perceptions, this appears to be the only method to attain realism.

\section{REFERENCES}

Burton, G., \& Turvey, M. T. (1990). Perceiving the lengths of rods that are held but not wielded. Ecological Psychology, 2, 295-324.

Carello, C., Fitzpatrick, P., Domaniewicz, I., Chan, T.-C., \& TurvEY, M. T. (1992). Effortful touch with minimal movement. Journal of Experimental Psychology: Human Perception \& Performance, 18, 290-302.

Chan, T.-C. (1995). The effect of density and diameter on haptic perception of rod length. Perception \& Psychophysics, 57, 778-786.

Chan, T.-C., \& Turvey, M. T. (1991). Perceiving the vertical distances of surfaces by means of a hand-held probe. Journal of Experimental Psychology: Human Perception \& Performance, 17, 347-358.

CutTing, J. E. (1993). Perceptual artifacts and phenomena: Gibson's role in the 20th century. In S. C. Masin (Ed.), Foundations of perceptual theory (pp. 231-260). New York: Elsevier, North-Holland.

Fitzpatrick, P., Carello, C., \& Turvey, M. T. (1994). Eigenvalues of the inertia tensor and exteroception by the "muscular sense." Neuroscience, 60, 551-568.

Gibson, J. J. (1933). Adaptation, after-effect and contrast in the perception of curved lines. Journal of Experimental Psychology, 16, 1-31.

GiBson, J. J. (1966). The senses considered as perceptual systems. Boston: Houghton Mifflin.

Gibson, J. J. (1979). The ecological approach to visual perception. Boston: Houghton Mifflin.

HoIsINGTON, L. B. (1920). On the non-visual perception of the length of lifted rods. American Journal of Psychology, 31, 144-146.

HollingwORTH, H. L. (1928). Psychology: Its fact and principles. New York: D. Appleton.

Kugler, P. N., \& TURVEY, M. T. (1987). Information, natural law, and the self-assembly of rhythmic movement. Hillsdale, NJ: Erlbaum. Lederman, S. J., Ganeshan, S. R., \& Ellis, R. E. (in press). Haptic perception of the extent of statically held rods. Journal of Experimental Psychology: Human Perception \& Performance.
Lee, D. N., Lishman, J. R., \& Thomson, J. A. (1982). Regulation of gait in long-jumping. Journal of Experimental Psychology: Human Perception \& Performance, 8, 448-459.

LeE, D. N., \& Reddish, P. E. (1981). Plummeting gannets: A paradigm of ecological optics. Nature, 293, 293-294.

MCKenna, F. P. (1984). Assimilation and contrast in perceptual judgments. Quarterly Journal of Experimental Psychology, 36A, 531548.

Solomon, H. Y., \& TuRvey, M. T. (1988). Haptically perceiving the distances reachable with hand-held objects. Journal of Experimental Psychology: Human Perception \& Performance, 14, 404-427.

Solomon, H. Y., Turvey, M. T., \& Burton, G. (1989). Perceiving extents of rods by wielding: Haptic diagonalization and decomposition of the inertia tensor. Journal of Experimental Psychology: Human Perception \& Performance, 15, 58-68.

Turvey, M. T., \& Carello, C. (1986). The ecological approach to perceiving-acting: A pictorial essay. Acta Psychologica, 63, 133155.

Turvey, M. T., Carello, C., \& Kim, N. G. (1990). Links between active perception and the control of action. In H. Haken \& M. Stadler (Eds.), Synergetics of cognition (pp. 269-295). Berlin: Springer-Verlag.

Turvey, M. T., Solomon, H. Y., \& Burton, G. (1989). An ecological analysis of knowing by wielding. Journal of the Experimental Analysis of Behavior, 52, 387-407.

WAGNER, H. (1982). Flow-field variables trigger landing in flies. $\mathrm{Na}$ ture, 297, 147-148.

WARREN, R. M. (1985). Criterion shift rule and perceptual homeostasis. Psychological Review, 92, 574-584.

WYszecki, G. ( 1986). Color appearance. In K. R. Boff, L. Kaufman, \& J. P. Thomas (Eds.), Handbook of perception and human performance: Vol. 1. Sensory processes and perception (pp. 9.1-9.57). New York: Wiley.

\section{NOTES}

1. In Experiment 5 of Carello et al. (1992), gravitational torque (or static torque) was found to be more accurate than first moment in predicting the perceived length. Static torque takes care of the orientation of the rod, while first moment does not. The static torque is a rotational tendency dependent on the mass distribution around the wrist as depicted by the first moment. It has the same value (in $\mathrm{gwt} \cdot \mathrm{cm}$ ) as the first moment (in $\mathrm{g} \cdot \mathrm{cm}$ ) when the rod is held horizontally. Although the turning tendency and the upward pull at the wrist can be transformed to two opposite noncollinear forces $\mathbf{U}$ and $\mathbf{D}, \mathbf{U}$ is an upward thrust and should not be mistaken as the static torque.

2. There are two scaling coefficients, $b$ and $p$, in the power law, $\psi(\lambda)=b x^{p}$, where $b$ is a linear multiplier and $p$ is the exponent. Usually, the power coefficient, $p$, is regarded as the parameter that characterizes the perceptual continuum and therefore should stay unchanged. Yet previous studies have shown that $p$ can vary a lot between conditions. It would be too involved here to determine the independent change of these two scaling coefficients. Thus, when I state that the scaling coefficients increase or are large. I mean that the equivalent result of the change of both coefficients produces a larger estimate.

3. $I_{\mathrm{xx}}$ is used here instead of $I_{1}$ and $I_{3}$, because the equation with $I_{1}$ and $I_{3}$ produced by Turvey and his colleagues differs in each case. It is uncertain what the value of the power coefficients should be. Since in Solomon and Turvey's (1988) Experiment 1 over $90 \%$ of the variance in perceived length is accounted for by $I_{x x}$, and since in Chan and Turvey's (1991) Experiment $398 \%$ of the variance in perceived distance is accounted for by $I_{x x}, I_{x x}$ can be regarded as the parameter good enough to specify perceived length when the rod is wielded. 
APPENDIX

Dynamic Variables Used in This Article

Variables With Definitions and Formulas Units

Weight (W): The gravitational pull on an object. $\mathbf{W}=m \mathbf{g}$, where $m$ is the mass, and $\mathbf{g}$ is the acceleration due to gravity. When expressed in units of gram weight, the value of $\mathbf{W}$ is the same as that for $m$.

First moment of mass (M): Distribution of mass with reference to an axis. $\mathbf{M}=m \mathbf{r}$ where $m$ is the mass of the rod and $\mathbf{r}$ is the moment arm.

Static torque $\left(\mathbf{N}_{\mathrm{s}}\right)$ : The turning tendency when the weight of the rod does not act through the grip. $\mathbf{N}_{\mathrm{s}}=\mathbf{W r}$, where $\mathbf{W}$ is the weight of a rod and $\mathbf{r}$ is the perpendicular distance from the center of the rod to the line passing through the grip. It is also called gravitational torque (Carello et al., 1992). It has the same value of first moment of mass (in $\mathrm{g} \cdot \mathrm{cm}$ ) if a rod is held horizontal.

Inertial torque $\left(\mathbf{N}_{\mathrm{i}}\right)$ : Rotational force needed to rotate a mass of certain moment of inertia $(I)$ at an angular acceleration $(\dot{\omega})$. The magnitude of the inertial torque can be obtained from the equation $\mathbf{N}_{\mathbf{i}}=I \dot{\omega}$.

Moment of inertia $(I)$ : Resistance to rotational acceleration of an object. For a cylindrical rod of mass, $m$, length, $L$, and radius, $a$, grasped at one end at a distance, $r$, from the center of mass with the wrist at a distance, $d$, from the center of the rod (see Figure 4), the moment of inertia about the axis through the wrist and parallel to $x$ is:

$$
I_{\mathrm{xx}}=(m / 12)\left(3 a^{2}+L^{2}\right)+m\left(r^{2}+d^{2}\right) .
$$

For a cylindrical rod, the moment of inertia $\left(I_{\mathrm{zz}}\right)$ about the $z$ axis through the wrist is similar to $I_{\mathrm{xx}}$.

$$
I_{\mathrm{zz}}=(m / 12)\left(3 a^{2}+L^{2}\right)+m r^{2} .
$$

The moment of inertia about the $y$ axis through the wrist is:

$$
\begin{aligned}
I_{\mathrm{yy}}= & m a^{2}+m d^{2} \text { for hollow rods, or } \\
& =m a^{2 / 2}+m d^{2} \text { for solid rods. }
\end{aligned}
$$

Inertia tensor: A property of the rod that characterizes the resistance of rotational acceleration of the rod in three dimensions. It is represented by a $3 \times 3$ matrix.

$$
\left[\begin{array}{lll}
I_{\mathrm{xx}} & I_{\mathrm{xy}} & I_{\mathrm{xz}} \\
I_{\mathrm{yx}} & I_{\mathrm{yy}} & I_{\mathrm{yz}} \\
I_{\mathrm{zx}} & I_{\mathrm{zy}} & I_{\mathrm{zz}}
\end{array}\right]
$$

(Manuscript received January 13, 1995; revision accepted for publication December 18,1995 .) 